\title{
Possible Risk Factors Associated with Relapse in Patients Treated with Neoadjuvant Chemohormonal Therapy for High Risk Prostate Cancer
}

\author{
R. S. Bhatt ${ }^{1}$, L. Werner ${ }^{2}$, M.M. Regan ${ }^{2}$, J. Yannucci ${ }^{3}$, Y-J, Ko ${ }^{4}$, H-Y Wang ${ }^{1}$, S. Rosen $^{6}$, E. Genega ${ }^{6}$, \\ M-E. Morrissey ${ }^{1}$, S. Duggan ${ }^{1}$, C Vazquez ${ }^{1}$, M. Sanda ${ }^{5}$, W. DeWolf ${ }^{5}$, S. Balk ${ }^{1}$, X. Yuan ${ }^{1}$ and G.J. Bubley ${ }^{1, *}$ \\ ${ }^{I}$ Division of Hematology-Oncology and Cancer Biology, Beth Israel Deaconess Medical Center, Harvard Medical \\ School, Boston MA 02215, USA \\ ${ }^{2}$ Department of Biostatistics and Computational Biology, Dana-Farber Cancer Institute; Harvard Medical School, \\ Boston MA 02215, USA \\ ${ }^{3}$ Low Country Cancer Care, Savannah, GE 31405, USA \\ ${ }^{4}$ Division of Oncology, Sunnybrook Regional Cancer Centre, Toronto, Ontario, Canada M4N 3 M5 \\ ${ }^{5}$ Division of Urology, Beth Israel Deaconess Medical Center, Harvard Medical School, Boston MA 02215, USA \\ ${ }^{6}$ Department of Pathology, Beth Israel Deaconess Medical Center, Harvard Medical School, Boston MA 02215, USA
}

\begin{abstract}
Objectives: To perform a pilot hypothesis generating study of neoadjuvant docetaxel, estramustine, and androgen deprivation therapy for high-risk patients prior to radical prostatectomy.

Patients and Methods: Twenty-eight patients received 4 cycles of docetaxel and estramustine administered on an every three week schedule in combination with androgen deprivation therapy (LHRH analog and bicalutamide) prior to radical prostatectomy. Following the prostatectomy, androgen deprivation therapy was continued for 8 months. End-points were pathological complete response (pCR), time-to-relapse (TTR), feasibility and tolerability, and biochemical or clinical correlates of relapse.

Results: With a median of 80 months (6.6 years) of follow-up, 18/28 patients have relapsed and one patient died from unrelated causes while in remission, with median TTR of 44 months (3.6 years). Tumor downstaging, perhaps as a result of neoadjuvant therapy, was associated with a decreased risk of relapse $(\mathrm{P}=0.0002)$. Consistent with this result, positive margin status was associated with an increased risk of relapse, which was not affected by adjuvant radiation therapy. Increased expression of a possible tumor stem cell marker, (Sry-related high mobility group box-9) Sox-9, both at the time of prostatectomy $(\mathrm{P}=0.005)$ and in the pre-treatment tumor biopsy cores was associated with an increased risk of relapse $(\mathrm{P}=0.03)$.

Conclusions: The neoadjuvant chemohormonal regimen may have benefited some patients, especially those who exhibited pathologic downstaging. Sox-9 expression in prostate cancer specimens warrants prospective validation in both pre-prostatectomy tissue as well as patients undergoing an ongoing randomized trial using a similar neoadjuvant regimen.
\end{abstract}

Keywords: Neoadjuvant, high-risk prostate cancer, Sox-9.

\section{INTRODUCTION}

Multiple studies have defined risk factors for recurrence and death from prostate cancer. The three independent parameters consistently identified are high clinical stage (>cT2), elevated PSA (>10) and high Gleason score (>7) [1]. In patients with one or more of these poor prognostic factors, the likelihood of cure from primary therapy (prostatectomy or radiation therapy) decreases significantly [2] and the management of high risk, localized prostate cancer remain uncertain.

*Address correcpondence to this author at the Division of Hematology Oncology and Cancer Biology, Beth Israel Deaconess Medical Center, Boston MA 02215, USA; Tel: 617-735-2062; Fax: 617-735-2060;

E-mail: gbubley@bidmc.harvard.edu
Androgen deprivation therapy (ADT) has proven to be a useful adjuvant therapy for men with high-stage prostate cancer treated with external beam radiotherapy (EBRT) [3, 4]. In contrast, ADT as neoadjuvant therapy in conjunction with radical prostatectomy (RP) has not improved survival [5-7]. Because docetaxel has been shown to result in a survival advantage for castrate resistant metastatic disease $[8,9]$ it has been given alone and with ADT in the neoadjuvant setting for high risk prostate cancer [10-17]. The rationale for combining chemotherapy and hormonal therapy is derived from tumor models that demonstrate that a combined approach is better than sequential therapy with either modality alone [16-18]. However, although neoadjuvant chemohormonal therapy has been shown to be safe [10-17], and sometimes has resulted in better than expected outcomes 
[15-17], consistent clinical or pathological markers associated with improved outcome have yet to be identified.

We performed a trial that combined ADT with docetaxel and estramustine for 4 [3] week cycles prior to radical prostatectomy (RP). ADT was continued post-RP for 8 months. Because PSA recurrence can be prolonged even in high risk prostate cancer, this cohort has had long-term follow-up. Goals were to correlate time-to-relapse (TTR) with clinical and biochemical parameters. In addition, we generated a tissue microarray from the RP specimens to assess the effect of expression of a number of conventional biomarkers, as well as a possible prostate cancer stem cell marker, Sox (Sry-related high-mobility group box)-9 [19-21].

Our interest in Sox-9 stems from previous work from our group that demonstrated that Sox-9 expression is significantly increased in recurrent castrate resistant prostate cancer compared to primary cancers [19]. Sox-9 is a transcription factor important in the development of male testes and is expressed in areas of the developing prostate associated with stem cell properties [19]. Furthermore, Sox-9 expression is correlated with higher Gleason grade disease [20]. While silencing endogenous Sox-9 expression in PCa xenografts causes reduced tumor growth, Sox-9 over-expression enhances PCa xenograft growth, angiogenesis and invasion [21]. These results suggest a potential role for Sox-9 in PCa aggressive behavior and possibly in the setting of treatment resistance.

\section{METHODS}

\section{Patients}

Between September 28, 1999 and May 17, 2005, 28 patients with high-risk localized prostate cancer were enrolled on this IRB approved phase II protocol. Eligible patients were required to demonstrate one or more of the following criteria: clinical stage (T3), elevated PSA $(\geq 20 \mathrm{ng} / \mathrm{ml})$ or high Gleason score $(>7)$. In addition patients with 5 of 6 positive core biopsies or the equivalent with Gleason $4+3$ disease were also eligible. Patients had to have normal renal, liver, cardiac and hematopoetic function, and no other active cancers, infection, or significant neuropathy. Endorectal MRI evaluation was not required.

\section{Staging Evaluation}

Patients were assigned a clinical stage according to the tumor-node-metastasis (TNM) staging system based on digital rectal examination (DRE). Clinical stage T3 or extraprostatic extension was assigned on the basis of a DRE performed by one Urologist (WCD) and confirmed by the study PI (GJB). Chest X-ray, CT of the abdomen and pelvis, and bone scans were required of all patients before study entry to exclude the presence of metastatic disease.

\section{Treatment}

Patients were treated with a combination regimen of 4 cycles (administered every 3 weeks) of docetaxel (70 $\mathrm{mg} / \mathrm{m} 2$ ) and estramustine (280 $\mathrm{mg}$ orally tid on days 1-5). An LHRH agonist and bicalutamide (50mg/day) were initiated one week prior to chemotherapy and continued for one year. Patients were treated with dexamethasone prior to docetaxel infusion and $1 \mathrm{mg}$ of warfarin was administered as prophylaxis for DVT through cycle 4 of chemotherapy.
Complete blood counts, liver function tests and serum PSAs were obtained on day 1 of each cycle. RP was performed 1619 weeks after beginning therapy.

\section{Pathology}

One of two pathologists (SR, EG) assessed pathological staging on post- prostatectomy specimens. Staging including a description of all tumor foci within the gland, presence or absence of perineural invasion and/or lymphovascular invasion, presence of extraprostatic extension of tumor (including seminal vesicle invasion), and margin status.

\section{Immunohistochemistry}

Immunohistochemistry (IHC) was performed as previously described for Sox-9, AR, and Ki-67 antibodies (18-20) or following manufacturer's recommendations using the following antibodies: rabbit anti-androgen receptor antibody (PG-21, and Cell Signaling \#3202;1:30); rabbit anti-phosphoHistone H3 (Ser10, 06-570:1:250, Upstate Biotechnologies); rabbit anti-Sox-9 (1:1500) (Dr. M. Wegner, Institut fur Biochemie, Emil-Fischer-Zentrum, Universitat Erlangen Erlangen, Germany); mouse anti-Ki67 (1:300) (a gift from Dr. S. Signoretti, DFCI, Boston, MA); Bcl2 (1:500, Cell Signaling 2870); p53 (1:500 Cell Signaling 2527); HIF1 $\alpha$ (Novus: $1: 200)$. IHC staining was performed on pre-treatment prostate cores biopsies and a TMA having 2 to 3 samples involved with cancer from different regions of the gland.

\section{Tissue Microarray Construction and Core Biopsy Analysis}

The paraffin blocks containing tumor were obtained from 23 of 28 patients in this study, and tissue blocks were designated as to their location within the prostate. In 18 cases, tumor for the TMA was obtained from the left and right lobe of the prostate. In 3 other cases, tumor was obtained from the apex and base. In two cases, tumor was derived from only one remaining focus of tumor. In 16 cases, three separate cores with tumor were obtained, in 4 patient samples two cores with tumor were obtained and three patients had only one core with tumor. Normal non-malignant tissue was available from three areas of the prostate in all 23 patients sampled.

Tissue was removed using a minute tissue cylinder $(0.6 \mathrm{~mm})$ by an experienced technician into areas of tumor and normal prostate and subsequently transferred into a premade hole at defined array coordinates into the recipient tissue block. The location for the tumor "punch" was guided by an $\mathrm{H} \& \mathrm{E}$ stained section overlaid on the surface of the donor block.

Additionally, 13 of 28 patients had pre-therapy core biopsies available for IHC analysis, 12 for which prostatectomy specimens were also available. These were not analyzed by TMA due to limitations of tissue depth. However, fresh cut slides from the blocks were cut and used for IHC analyses.

\section{Follow-Up}

Patients were evaluated 1 month after surgery, then at 3 month intervals by history, physical examination, and routine laboratory tests including PSA. 
Patients with positive surgical margins or seminal vesicle involvement were treated with adjuvant radiation therapy at the discretion of the treating physician. Following PSA relapse, patients were treated with salvage radiation therapy if they had not already undergone adjuvant radiation therapy.

\section{Statistical Considerations}

The primary objective of the study was to determine the pCR rate and TTR for the cohort. Secondary objectives of this study were to assess overall survival, feasibility and toxicity. Correlative aims were to identify possible clinical or IHC markers that predicted for relapse.

Descriptive statistics summarized clinical characteristics either as number and percent or as median and interquartile range of values. Relapse was defined as a confirmed postRP PSA rising to a level $>0.2 \mathrm{ng} / \mathrm{ml}$ or the need for an additional therapy (other than adjuvant treatment), whichever came first. One patient who died from an unrelated cause before relapse occurred was censored at the last PSA date prior to death. TTR was summarized using KaplanMeier method and comparisons were made using a log rank test.

To assess the relevance of a change between clinical and pathological staging, patients were categorized as upstaged, downstaged or not changed using the T-stage. Each protein assessed by IHC was categorized into high and low staining, where high was $75 \%$ of cells staining at an intensity of $3+$. The tumor core within the TMA or core biopsy sample with the highest staining characteristics was used to assess each patient.

\section{RESULTS}

\section{Patient Characteristics}

The clinical characteristics of the study population are summarized in Table 1. The Gleason score was $>7$ in 18 of 28 patients and one patient was not assigned a Gleason score based on a poorly differentiated ductal histology. Eight (29\%) patients had PSA values between 10 and $20 \mathrm{ng} / \mathrm{ml}$ and 9 had PSA values of $>20 \mathrm{ng} / \mathrm{ml}(32 \%)$. Two (7\%) patients were clinical stage T1c (Gleason 8 and Gleason 9 scores). Three patients were clinical stage T2 (1 with a PSA of 32 $\mathrm{ng} / \mathrm{ml}$ and 1 with Gleason 8) and $23(82 \%)$ patients were clinical stage T3. One T2 patient was enrolled who did not meet the PSA criteria of $>20 \mathrm{ng} / \mathrm{ml}$, T3 clinical stage or Gleason score $>7$, this patient had 6 of 6 positive cores with Gleason $4+3$ disease. Many of the clinical stage T3 disease patients also exhibited high PSA values and Gleason scores $>7$ (Table 1).

\section{Feasibility/Toxicity}

All 28 patients completed 4 cycles of neoadjuvant chemotherapy and underwent RP. There were no unanticipated adverse events from chemo-hormonal therapy, although 4 patients experienced fever and neutropenia and 1 developed a grade 3 elevation of transaminases that normalized after discontinuation of bicalutamide therapy. Following RP, two (7\%) patients suffered protracted ileus that prolonged their hospitalization, one patient developed pancreatitis, and one developed a pulmonary embolus. One-half (14/28) of the patients required $\mathrm{RBC}$ transfusions post RP.
Table 1. Patient and Disease Characteristics at Baseline

\begin{tabular}{|c|c|c|}
\hline & \multicolumn{2}{|c|}{$\begin{array}{l}\mathrm{N} \text { and } \% \text { or Median } \\
\text { and IQR }\end{array}$} \\
\hline Number of Patients & 28 & $100 \%$ \\
\hline Age at Enrollment (years), median and IQR & 57 & $53-64.5$ \\
\hline PSA at Enrollment (ng/ml), median and IQR & 14 & $6.5-33$ \\
\hline $\mathrm{PSA} \leq 4 \mathrm{ng} / \mathrm{ml}$ & 2 & $7 \%$ \\
\hline $4 \mathrm{ng} / \mathrm{ml}<\mathrm{PSA} \leq 10 \mathrm{ng} / \mathrm{ml}$ & 9 & $32 \%$ \\
\hline $10 \mathrm{ng} / \mathrm{ml}<\mathrm{PSA} \leq 20 \mathrm{ng} / \mathrm{ml}$ & 8 & $29 \%$ \\
\hline $\mathrm{PSA}>20 \mathrm{ng} / \mathrm{ml}$ & 9 & $32 \%$ \\
\hline \multicolumn{3}{|l|}{ Clinical stage } \\
\hline $\mathrm{T} 1$ & 2 & $7 \%$ \\
\hline $\mathrm{T} 2$ & 3 & $11 \%$ \\
\hline $\mathrm{T} 3$ & 23 & $82 \%$ \\
\hline \multicolumn{3}{|l|}{ Biopsy Gleason Score } \\
\hline 6 & 2 & $7 \%$ \\
\hline $7(3+4)$ & 2 & $7 \%$ \\
\hline $7(4+3)$ & 5 & $19 \%$ \\
\hline 8 & 8 & $30 \%$ \\
\hline 9 & 10 & $37 \%$ \\
\hline Unknown & 1 & \\
\hline \multicolumn{3}{|l|}{ Race } \\
\hline Caucasian & 27 & $96 \%$ \\
\hline Black & 1 & $4 \%$ \\
\hline
\end{tabular}

\section{Clinical Characteristics and Outcome}

Table 2 shows the PSA level prior to surgery, the pathological staging after RP, and the type of radiation therapy administered after RP. All patients had undetectable PSA levels post-operatively. Approximately half the patients had an undetectable PSA prior to RP.

Pathological assessment of RP specimens demonstrated that $10(36 \%)$ patients had carcinoma confined to the prostate, $15(54 \%)$ had evidence of extraprostatic extension or seminal vesicle invasion and $3(11 \%)$ had evidence of bladder invasion. Three $(11 \%)$ patients had microscopically positive lymph nodes.

The median TTR is 44 months (3.6 years), and 18 of 28 patients relapsed with a median follow up of 80 months $(6.6$ years) (Fig. 1A). Nine patients remain free of PSA relapse, and one patient died from unrelated causes while still relapse-free. Of the 18 relapsed patients, 9 were treated with salvage radiation therapy (Table 2) and 4 of these patients currently demonstrate undetectable PSA values for a median of 29 months from the completion of salvage radiation therapy. Thus with 6.6 years of follow-up, 13/28 patients have undetectable PSA values. 
Table 2. PSA Prior to Surgery, Pathological Staging and Adjuvant Radiation Therapy after Surgery

\begin{tabular}{|c|c|c|}
\hline & $\mathbf{N}$ & $\%$ \\
\hline \multicolumn{3}{|l|}{ PSA Prior to Surgery (ng/ml) } \\
\hline PSA $<0.3 \mathrm{ng} / \mathrm{ml}$ & 15 & 54 \\
\hline $\mathrm{PSA} \geq 0.3 \mathrm{ng} / \mathrm{ml}$ & 13 & 46 \\
\hline \multicolumn{3}{|l|}{ Pathological Staging } \\
\hline \multicolumn{3}{|l|}{ T-stage } \\
\hline $\mathrm{T} 2$ & 10 & 36 \\
\hline $\mathrm{T} 3$ & 15 & 54 \\
\hline $\mathrm{T} 4$ & 3 & 11 \\
\hline N1 & 3 & 11 \\
\hline Positive Margins & 11 & 39 \\
\hline Adjuvant XRT & 8 & 29 \\
\hline Salvage XRT & 9 & 32 \\
\hline
\end{tabular}

Of the 14 relapsed patients, 2 patients have not required any therapy, 8 are responding to ADT and 4 have developed castrate resistant disease. Of these, 2 have died from prostate cancer, 2 are being treated with docetaxel and another with salvage hormonal therapy. For the 5 deceased patients, 4 had PSA relapse, 2 died of prostate cancer, 1 of lung cancer and 1 of cardiac disease. One patient died while in remission from pancreatic cancer 6.2 years after RP.

The association of PSA values on TTR was evaluated both for PSA levels at enrollment and PSA values prior to RP. Entry PSA levels $>10 \mathrm{ng} / \mathrm{ml}$ were a risk factor for relapse (Fig. 1B) $(\mathrm{P}=0.032)$. Patients that had undetectable PSA levels prior to surgery $(15 / 28)$ had a slight improvement in TTR, but this was not significant $(\mathrm{P}=0.055)$. Interestingly, patients older than the median had a significantly better TTR (Fig. 1C, $\mathrm{P}=0.016$ ).

\section{Pathological Characteristics and Overall Outcome}

We assessed the association of pathological characteristics and TTR. There was a strong association with relapse comparing 18 pT3 and pT4 patients to 10 pT2 patients (Fig. $\mathbf{2 A}, \mathrm{P}=0.002)$. In contrast, clinical stage was not associated with RFS, however, this was not surprising as $>80 \%$ of patients were categorized as clinical stage T3.

Positive surgical margins (11/28 patients) were associated with increased risk of relapse (Fig. 2B, $\mathrm{P}=0.0002)$. Although 7 of 8 patients with positive margins and negative nodes were treated with adjuvant radiation therapy, relapse was not prevented for any of these patients.

Although neoadjuvant chemohormonal therapy did not result in pathologic complete responses, there were 7 cases in which the patient had a lower pathologic stage than the clinical stage and of those, 6 are currently relapse free (Fig. 2C, $\mathrm{P}=0.017$ ). In contrast, of the 18 patients who relapsed, only one patient was downstaged at surgery, and 11 patients were upstaged at the time of surgery (from T1c to T2c-T4) and 9 have relapsed.

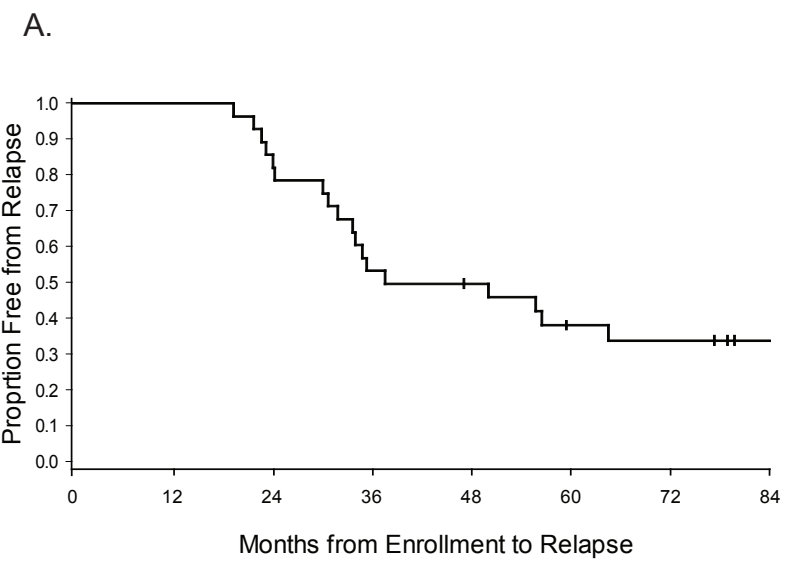

B.

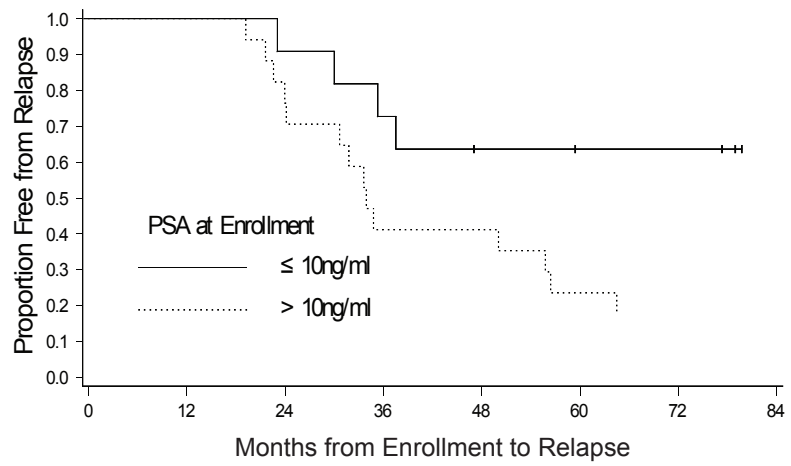

C.

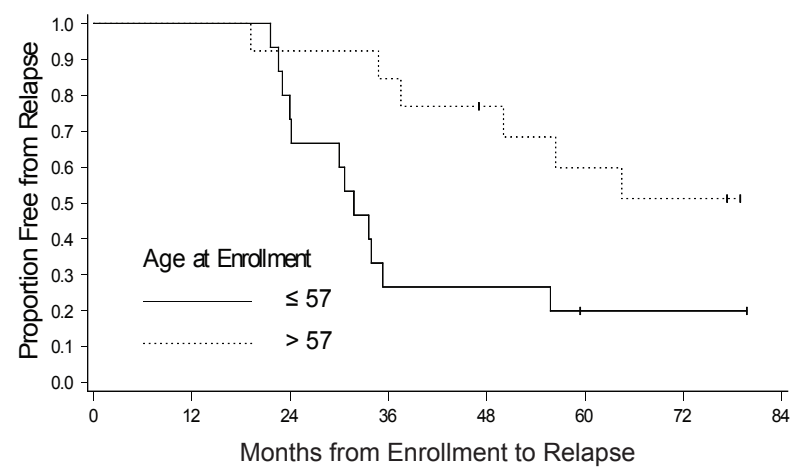

Fig. (1). Kaplan Meier estimate of time to relapse (TTR) for the entire cohort (A), by PSA at enrollment (B) and by age (C) at enrollment. 1A. Entire Cohort: Eighteen patients have relapsed with a median follow up of 80 months (6.6 years). Median time to relapse is 44 months. 1B. By PSA at enrollment: PSA levels greater than $10 \mathrm{ng} / \mathrm{ml}$ at diagnosis were associated with an increased risk for relapse $(\mathrm{P}=0.032)$. 1C. By age: Age $\leq 57$ years at enrollment was associated with an increased risk of relapse $(\mathrm{P}=0.016)$.

\section{Immunohistochemical Analysis and Outcome}

IHC analysis on RP specimens was available for 23 of 28 patients. The remaining 5 patients had residual volume too 
small to use for tumor microarray analysis. Of these, 4 have not relapsed.

A.

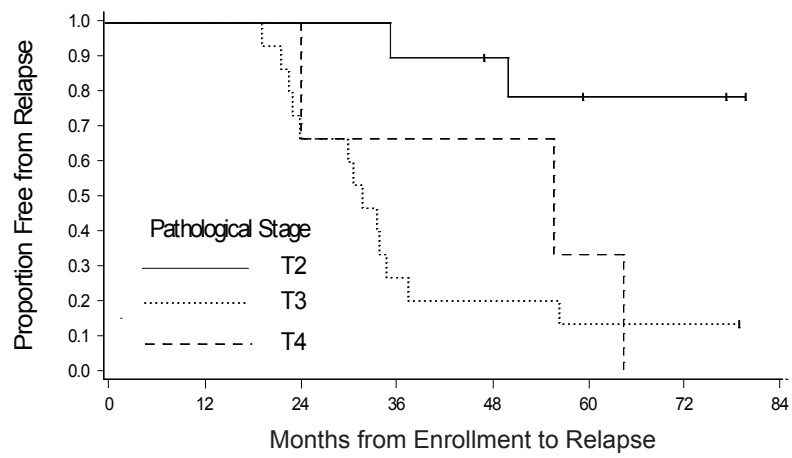

B.

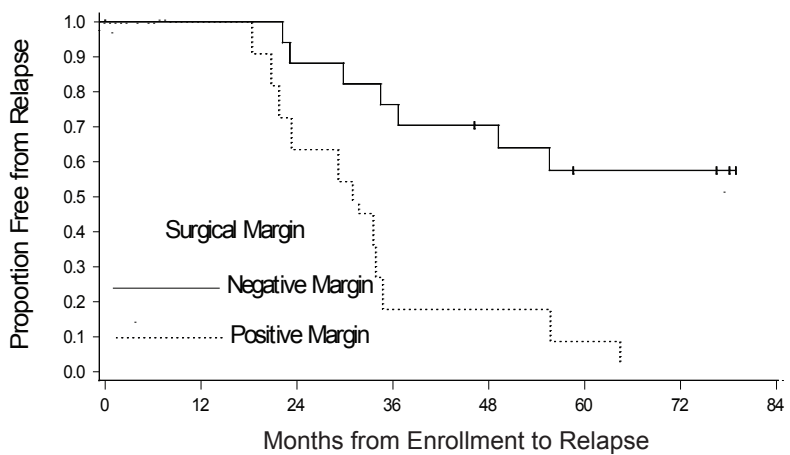

C.

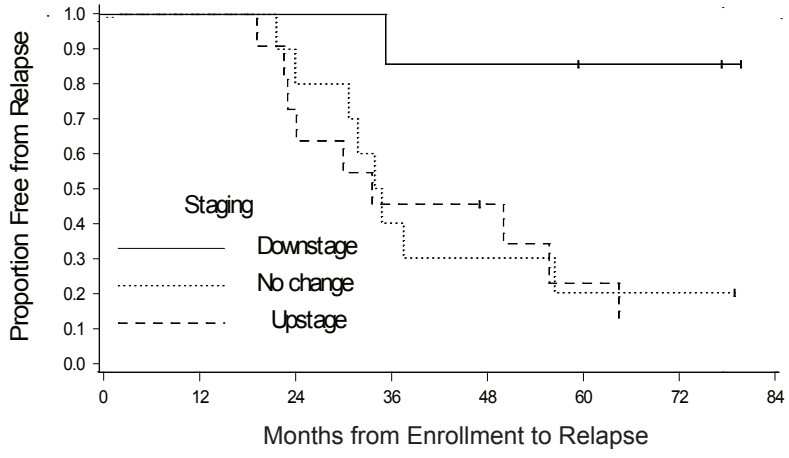

Fig. (2). Kaplan Meier estimate of time to relapse by pathologic $T$ stage (2A), surgical margin status (2B), and by comparisons between clinical and pathological staging (2C). 2A. Pathologic stage Patients with pathologic T3 and T4 disease $(n=18)$ had a significantly increased risk for relapse in comparison to pathologic T2 patients $(\mathrm{n}=10)(\mathrm{P}=0.002)$. 2B. Tumor margins: Positive tumor margins $(n=11)$ were associated with an increased risk for relapse $(\mathrm{P}=0.0002)$. 2C. Tumor downstaging: Downstaging from clinical stage to pathologic stage was associated with a decreased risk for relapse $(n=6 / 7)(p=0.017)$

We assessed IHC markers of proliferation (Ki-67 and phospho-histone H3), apoptosis (Bcl-2), as well as HIF-1 $\alpha$, mutant p53, AR and Sox-9 levels on the TMA tumor samples derived from the RP specimens. Of all the makers ana- lyzed, only Sox-9 expression was associated with TTR (Fig. 3A, $\mathrm{P}=0.005$ ). In normal prostate epithelium, Sox-9 expression is restricted to basal cells, but is present in luminal cells in prostate cancer (Fig. 4, [20, 21]).

Based on the observation that high Sox-9 expression in RP samples was associated with a decrease in TTR, we subsequently obtained pre-treatment core biopsies on 13 of the 28 patients. Only 1 of 6 patients with low Sox-9 expression in their core biopsy samples relapsed. In contrast, 6 of 7 patients with high Sox -9 expression relapsed $(p=0.03)$ (Fig. 3B).

\section{DISCUSSION}

An important asset of the current study is that it has one of the longest median follow-up periods reported ( 80 months or 6.6 years) to date, and additional relapses beyond the $18 / 28(64 \%)$ that have relapsed, are unexpected. Among the relapsed patients, 4 are in remission following salvage radiation therapy, making $13 / 28$ in complete remission. These data compare favorably to other neoadjuvant chemohormonal trials. Similar treatment regimens demonstrated a $58 \%$ relapse rate (53 month follow-up) (15) and a $55 \%$ relapse rate (29 month follow up) [11]. Nonetheless the efficacy of this treatment in a single arm study is not certain and comparisons between these and other neoadjuvant studies [5-7, 22-26] are problematic due to differences in regimens, duration of therapy and patient selection.

In this study, patients who exhibited pathological downstaging had a statistically significant improvement in outcome. Among the 7 patients downstaged, only one relapsed. Alternatively, in the group of 18 patients who relapsed, only one patient was downstaged at RP. Although this could reflect inaccuracies of clinical staging, this is unlikely, since the same urologist performed all clinical staging. Therefore one hypothesis from these data is that unlike neoadjuvant ADT alone, downstaging as reflected by pathological stage may reflect the sensitivity of microscopic cancer to chemohormonal therapy. Interestingly, neoadjuvant chemohormonal studies have been associated with greater than expected downstaging. In one neoadjuvant chemo-hormonal study, half the patients had organ-confined disease at RP, although only $8 \%$ would have been expected to, based on pre-operative Kattan nomogram assessment [15]. Furthermore, patients with pathologic complete remissions (pT0) disease have been detected in other docetaxel-based neoadjuvant studies $[14,15]$.

An important goal of this study was to analyze the molecular characteristics of residual cancer in RP specimens. Among a large number of IHC tumor makers analyzed in RP specimens, only Sox-9 expression was associated with significant risk of relapse. Given this finding, it was important to determine if Sox-9 expressing tumor cells present prior to therapy affected the outcome or if sox- 9 expression might be a marker of chemo-hormonal resistance. Towards that end, we were able to obtain a number of core pre-treatment core biopsy samples from the patients in this study. Analysis of these samples showed that high Sox-9 expression was statistically associated with relapse, suggesting that Sox-9 expression is a marker of intrinsic chemo-hormonal resistance, and less likely that cells expressing this protein are selected for 
A.

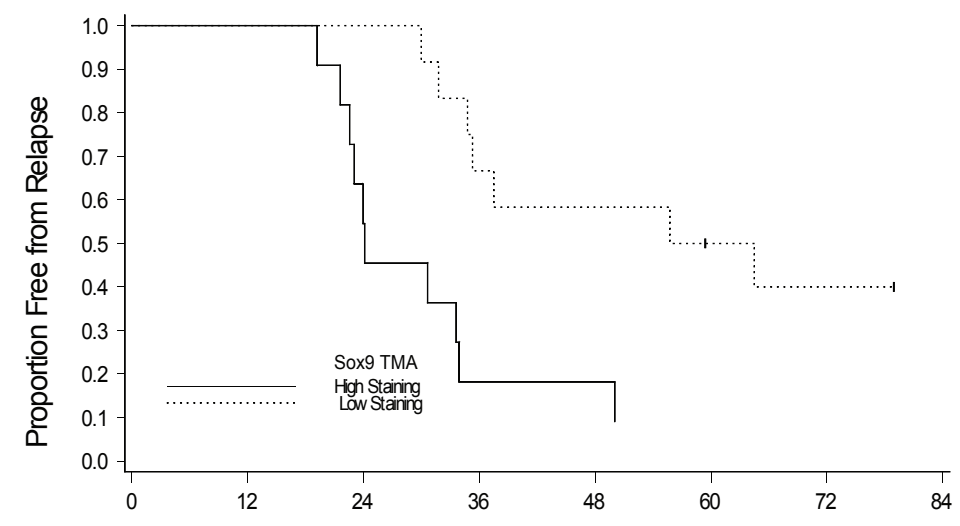

Months from Enrollment to Relapse

B.

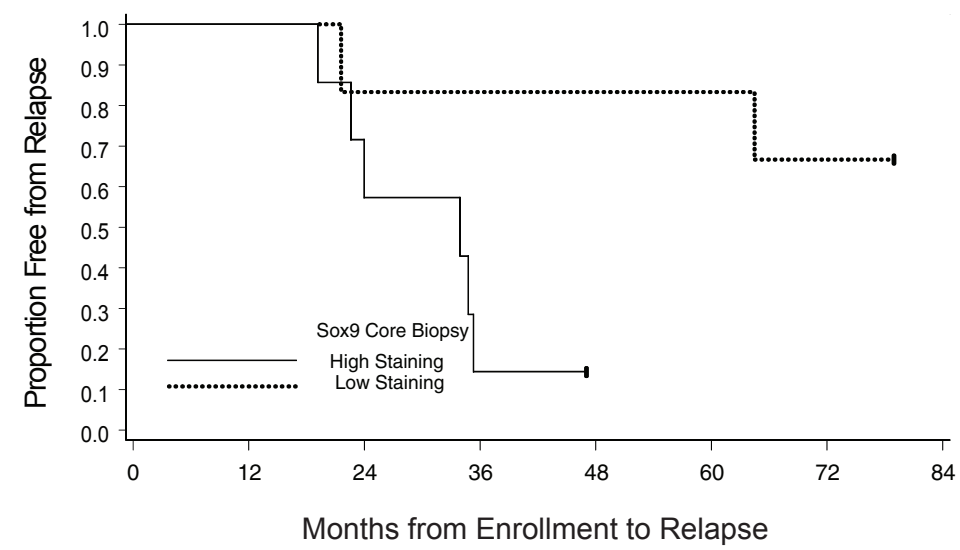

Fig. (3). Kaplan Meier Estimate of Time to Relapse by High and Low Sox-9 Staining. A. TMA sample analysis: High level of Sox-9 staining was associated with increased risk of relapse $(\mathrm{P}=0.005)(\mathrm{n}=11$ for high Sox-9 and 12 for low Sox-9). B. Core biopsy analysis. High Sox-9 expression in the tumor core biopsies $(n=7)$ was associated with increased risk of relapse compared to patients with low Sox-9 expression $(\mathrm{n}=6)$ (33.9 months vs TTP not reached, $\mathrm{P}=0.03)$.
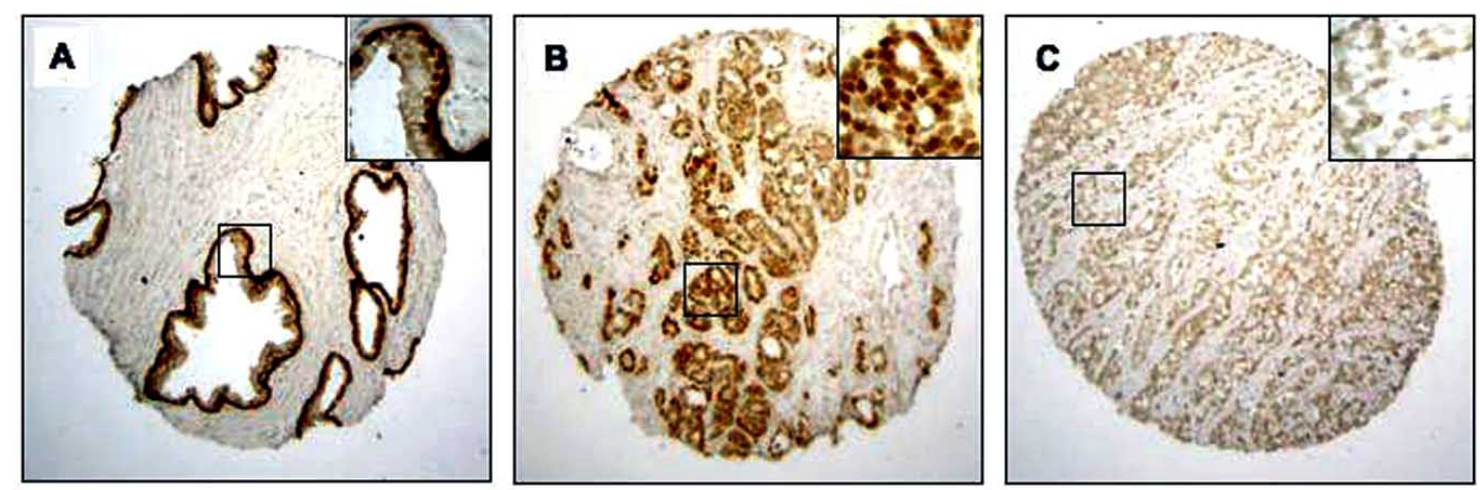

Fig. (4). Sox-9 expression in RP tissue microarray (TMA) specimens. A. Normal prostate glands B. Cancer glands with high Sox-9 expression. C. Cancer gland with low levels of Sox-9 expression. Depicted are images with 10X magnification and 20X for insets.

on the basis of resistance to therapy. These data suggest that Sox-9 expression may be a useful marker for future studies.

The biological significance of Sox-9 in prostate cancer is currently under investigation. Sox-9 levels are higher in castrate resistant prostate cancer has and Sox-9 has also been shown to play a role in the propagation of prostate cancer tumor stem cells, as well as invasion and angiogenesis [21].

Other clinical and pathological markers were somewhat informative. Not surprisingly, higher pretreatment PSA values were associated with an increased risk of relapse, 
likely reflecting tumor burden. An interesting finding was that patients older than the median age had a better outcome. Finally, 7 of 8 patients with positive margins relapsed despite the use of adjuvant radiation therapy and uninvolved lymph nodes, despite recent data that this therapy is useful for patients with positive margins [27]. Salvage XRT was, however, successful in 4 of 9 patients.

There are multiple unknowns associated with the optimal regimen of neoadjuvant chemo-hormonal therapy for prostate cancer. We used a docetaxel/estramustine regimen and schedule associated with a survival advantage in metastatic castrate resistant patients [8], but the value of estramustine is uncertain [9]. Identifying effective neoadjuvant strategies for high-risk prostate cancer is important, although this has been problematic and slow-going compared to strategies that have evolved for other solid tumors. Problems such as physician bias, the competing strategy of neoadjuvant hormonal therapy with EBRT, the development of newer androgen ablative therapies [28], and the length of follow up required to study survival, have made completing these trials a difficult task. Important also in future and ongoing trials will be the identification of factors associated with response. An important of this asset of this study is the identification of Sox-9 expression and degree of down-staging are potentially important parameters to be analyzed. These might be critically important for analysis in the ongoing randomized trial, CALGB 90203 [22] of neoadjuvant ADT and docetaxel prior to RP for high risk prostate cancer patients, similar to the patients included in this trial.

\section{CONCLUSIONS}

The current hypothesis generating trial of neoadjuvant chemohormonal regimen detected pathologic downstaging and Sox-9 expression as factors that may warrant prospective validation for patients undergoing these types of experimental therapies. Long term randomized neoadjuvant trials of this combination are necessary to establish this as a useful strategy, but these efforts should be combined with the identification of predictive clinical and biomarkers

NCT Trial Registration Number: \#NCT01230717

\section{ABBREVIATIONS}

$\begin{array}{lll}\text { ADT } & = & \text { Androgen Deprivation Therapy } \\ \text { EBRT } & = & \text { External Beam Radiation Therapy } \\ \text { IHC } & = & \text { Immunohistochemistry } \\ \text { LHRH } & = & \text { Leutinizing Hormone Releasing Hormone } \\ \text { pCR } & = & \text { Pathologic Complete Response } \\ \text { PSA } & = & \text { Prostate Specific Antigen } \\ \text { RP } & = & \text { Radical Prostatectomy } \\ \text { Sox-9 } & = & \text { Sry-related high-mobility group box-9 } \\ \text { TMA } & = & \text { Tissue MicroArray } \\ \text { TTR } & = & \text { Time to Relapse }\end{array}$

\section{ACKNOWLEDGEMENTS}

The authors wish to thank Dr. Sabina Signoretti with help in providing antibodies and in photography, Dr. Massimo Loda in construction of the tissue microassay and Dr. Isabel
Sesterhan for help with pathological preparation. We also wish to thank Sonal Bhatt for help with preparation of the manuscript.

\section{GRANT INFORMATION}

Hershey Family Foundation for Prostate Cancer Research and the Clinical Investigator Training Program: Beth Israel Deaconess Medical Center - Harvard/MIT Health Sciences and Technology, in collaboration with Pfizer Inc. and Merck \& Co (RSB)

\section{REFERENCES}

[1] D'Amico AV, Whittington R, Malkowicz SB, et al. A multivariate analysis of clinical and pathological factors that predict for prostate specific antigen failure after radical prostatectomy for prostate cancer. J Urol 1995; 154: 131

[2] Kattan MW, Eastham JA, Stapleton AM, et al. A preoperative nomogram for disease recurrence following radical prostatectomy for prostate cancer. J Natl Cancer Inst 1998; 90: 766.

[3] Pilepich, MV, Winter, K, Lawton, CA, et al. Androgen suppression adjuvant to definitive radiotherapy in prostate carcinoma-long-term results of phase III RTOG 85-31. Int J Radiat Oncol Biol Phys 2005; 61:1285.

[4] Bolla M, Collette L, Blank L, et al. Long-term results with immediate androgen suppression and external irradiation in patients with locally advanced prostate cancer (an EORTC study): a phase III randomized trial. Lancet 2002; 360: 103.

[5] Klotz LH, Goldenberg SL, Jewett MA, et al. Long-term followup of a randomized trial of 0 versus 3 months of neoadjuvant androgen ablation before radical prostatectomy. J Urol 2003; 170: 791.

[6] Gleave ME, Goldenberg L, Chin JL, et al. Randomized comparative study of 3 versus 8 months of neoadjuvant hormone therapy before radical prostatectomy: biochemical and pathological effects. J Urol 2001; 166: 500-507.

[7] Shelly MD, Kumar S, Wilt T, et al. A systematic review of metaanalysis of randomized trials of neo-adjuvant hormone therapy for localized and locally advanced prostate carcinoma Cancer Treat Rev 2009; 35: 9.

[8] Petrylak DP, Tangen CM, Hussain MH, et al. Docetaxel and estramustine compared with mitoxantrone and prednisone for advanced refractory prostate cancer. N Engl J Med 2004; 351: 1513.

[9] Tannock IF, de Wit R, Berry WR, et al. Docetaxel plus prednisone or mitoxantrone plus prednisone for advanced prostate cancer. $\mathrm{N}$ Engl J Med 2004; 351: 1502.

[10] Clark PE, Peereboom DM, Dreicer R, et al. Phase II trial of neoadjuvant estramustine and etoposide plus radical prostatectomy for locally advanced prostate cancer. Urology 2001; 57: 281.

[11] Konety BR, Eastham JA, Reuter VE, et al. Feasibility of radical prostatectomy after neoadjuvant chemohormonal therapy for patients with high risk or locally advanced prostate cancer: results of a phase I/II study. J Urol 2004; 171: 709.

[12] Mathew P, Pisters LL, Wood CG, et al. Neoadjuvant platelet derived growth factor receptor inhibitor therapy combined with docetaxel and androgen ablation for high risk localized prostate cancer. J Urol 2009; 181: 81.

[13] Pettaway, CA, Pisters, LL, Troncoso P, et al. Neoadjuvant chemotherapy and hormonal therapy followed by radical prostatectomy: feasibility and preliminary results. J Clin Oncol 2000; 18:1050.

[14] Chi KN, Chin JL, Winquist E, et al. Multicenter Phase II study of combined neoadjuvant docetaxel and hormone therapy before radical prostatectomy for patients with high risk localized prostate cancer. J Urol 2008; 180: 565.

[15] Prayer-Galetti T, Sacco E, Pagano F, et al. Long-term follow-up of a neoadjuvant chemohormonal trial before radical prostatecotmy in patients with non-metastatic high-risk prostate cancer. BJU 2007; 100: 274 .

[16] Sella A, Zisman A, Kovel S, et al. Neoadjuvant chemohormonal therapy in poor-prognosis localized prostate cancer. Oncology 2007; 71: 323 .

[17] Hussain M, Smith DC, El-Rayes BF, et al. Neoadjuvant docetaxel and estramustine chemotherapy in high-risk/locally advanced prostate cancer. Urology 2003; 61:774.

[18] Eigl BJ, Eggener SE, Baybik J, et al. Timing is everything: preclinical evidence supporting simultaneous rather than sequential 
chemohormonal therapy for prostate cancer. Clin Can Res 2005; 11: 4905 .

[19] Wang H, McKnight N, Zhang T, et al. SOX9 is expressed in normal prostate basal cells and regulates androgen receptor expression Cancer Res 2007; 67: 528.

[20] Thomsen MK, Ambroisine L, Wynn S, et al. SOX9 elevation in the prostate promotes proliferation and cooperates with PTEN loss to drive tumor formation. Cancer Res 2010; 70(3): 979-87.

[21] Wang H, Leav I, Ibargi S, et al. SOX9 is expressed in human fetal prosate epithelium and enhances prostate cancer invasion. Cancer Res 2008; 68: 1625

[22] Eastham JA, Kelly WK, Grossfield GD, et al. Cancer and Leukemia Group B (CALGB) 90203: a randomized phase 3 study of radical prostatectomy alone versus estramustine and docetaxel before radical prostatectomy for patients with high risk localized disease. Urology 2003; 62: 52.

[23] Soloway MS, Areek P, Sharifi K, et al. Neoadjuvant androgen ablation before radical prostatectomy in cT2bNxMo prostate cancer: 5-year results. J Urol 2002; 167: 112.
[24] Febbo PG, Richie JP, George DJ, et al. Neoadjuvant docetaxel before radical prostatectomy in patients with high-risk localized prostate cancer. Clin Cancer Res 2005; 11: 5233.

[25] Shepard DR, Dreicer R, Garcia J, et al. Phase II trial of neoadjuvant nab-paclitaxel in high risk patients with prostate cancer undergoing radical prostatectomy. J Urol 2009; 181: 1672.

[26] Van Poppel H, De Ridder D, Elgamal AA, et al. Neoadjuvant hormonal therapy before radical prostatectomy decreases the number of positive surgical margins in stage $\mathrm{T} 2$ prostate cancer: interim results of a prospective randomized trial. The Belgian UroOncological Study Group. J Urol 1995; 154: 429.

[27] Thompson IM, Tangen CM, Paradelo J, et al. Adjuvant radiotherapy for pathological T3N0M0 prostate cancer reduces the risk of metastases and improves long-term survival L long-term followup of a randomized clinical trial. J Urol 2009; 181: 956.

[28] Attard G, Reid AH, Olmost D, et al. Anti-tumor activity with CYP17 blockade indicates that castration-resistant prostate cancer frequently remains hormone driven. Cancer Res 2009; 69: 4937.

Received: December 14, 2010

Revised: January 21, 2011

Accepted: January 27, 2011

(C) Bhatt et al.; Licensee Bentham Open.

This is an open access article licensed under the terms of the Creative Commons Attribution Non-Commercial License (http://creativecommons.org/licenses/ by-nc/3.0/) which permits unrestricted, non-commercial use, distribution and reproduction in any medium, provided the work is properly cited. 